\title{
Investigating Field Efficacy and Safety of Conjunctival Brucella abortus S19 Vaccine in Cattle
}

\author{
S. Erdenlig Gurbilek ${ }^{1}$ M.S. Karagul', A.M. Saytekin¹, E.A. Baklan ${ }^{3}$, G. Saglam
}

10.18805/ag.DF-393

\begin{abstract}
Background: Vaccination is the most fundamental strategy in the control and eradication of brucellosis. Several vaccination programs with different vaccines have been carried out in many countries in which brucellosis continues to be a problem in livestock. One of the recommended vaccines against brucellosis in cattle is the live Brucella abortus S19 vaccine. The aim of this study is to evaluate the results of field safety and efficacy trials for the conjunctival Brucella abortus S19 vaccine prior to the mass vaccination program. Methods: In this study, 81 female cattle were vaccinated with a reduced dose of Brucella abortus S19 vaccine with the conjunctival route. The immune response after vaccination was investigated by suggested serological tests; namely, Rose Bengal Plate Test, Serum Agglutination Test and Complement Fixation Test.

Result: No adverse effect was observed within the scope of safety. Isolation of vaccine strain was observed only in a milk sample of lactating animals. Excluding the diagnosis criteria of the serological tests, humoral immune response was observed in most of the animals by all the serological tests one month after vaccination. Antibody levels lasted approximately 4 months after vaccination. In conclusion, the results of this study demonstrated that besides vaccine-induced antibodies, the vaccine including changes in dose and administration way in this study did not cause any significant risks for the target animals.

Key words: Brucella abortus, Cattle, Conjunctival, Efficacy, S19 vaccine, Safety.
\end{abstract}

\section{INTRODUCTION}

Brucellosis is a common zoonosis worldwide, which is caused by Brucella (B.) spp. (Godfroid et al., 2005; Songer and Post 2012; Yumuk and O'Callaghan 2012; Warreth, 2019). The disease is endemic and therefore, poses important risks in the Mediterranean countries (Warreth, 2019). Brucellosis in cattle usually occurs due to the B.abortus; however, $B$. melitensis and $B$. suis might be other causes of the disease (OIE, 2018a; Naveen Kumar et al., 2019). In the control of brucellosis, three main strategies; namely, hygienic practices, test-and-slaughter programs and vaccination are recommended (Nicoletti, 2010). Vaccination of all the susceptible hosts is the most effective way particularly in the areas with high prevalence (Briones et al., 2001). B. abortus $\mathrm{S} 19$ vaccine, the first effective vaccine, is commonly used against brucellosis in cattle (Schurig et al., 2002; Das et al., 2019; Ravi Kumar et al., 2019) and it is also compared with other vaccines as a reference vaccine (OIE 2018a). However, it leads to some possible complications such as abortion in pregnant cattle (Jimenes de Bagues et al., 1991; Schurig et al., 2002; Lalsiamthara and Lee 2017).

Based on the colonization of the regional lymph nodes by vaccine strain and low serological response, the reduced dose $\left(5 \times 10^{9}\right.$ colony-forming unit; cfu) of conjunctival S19 vaccine was recommended as the most proper vaccine for the vaccination of calves and cows (Plommet and Plommet, 1976). Testing for field safety and efficacy is required for the veterinary vaccines before they are administered to animals (OIE, 2018b).

In Turkey, several prevalence studies were carried out in the past in order to decide which vaccination strategy is
${ }^{1}$ Harran University, Faculty of Veterinary Medicine, Sanlıurfa, Turkey. ${ }^{2}$ Kartepe Vocational School of Equine Science, Kocaeli University, Kartepe, Kocaeli, Turkey.

${ }^{3}$ Pendik Veterinary Control Institute, Istanbul, Turkey. ${ }^{4}$ Samsun Veterinary Control Institute, Samsun, Turkey.

Corresponding Author: M.S. Karagul, Kartepe Vocational School of Equine Science, Kocaeli University, Kartepe, Kocaeli, Turkey. Email: msencerk@hotmail.com

How to cite this article: Gurbilek, S.E., Karagul, M.S., Saytekin, A.M., Baklan, E.A. and Saglam, G. (2022). Investigating Field Efficacy and Safety of Conjunctival Brucella abortus S19 Vaccine in Cattle. Agricultural Science Digest. DOI: 10.18805/ag.DF-393.

Submitted: 04-08-2021 Accepted: 15-12-2021 Online: 16-01-2022

needed to be implemented. In 2011 a comprehensive serological survey results showed that the herd prevalence rate was $(7.8 \%)$ in cattle and $(22.5 \%)$ in sheep. According to these quite high figures of herd prevalence, The Ministry of Agriculture and Forestry launched a new vaccination strategy, in which mass vaccination for all female cattle older than 3 months old was vaccinated by $B$. abortus $S 19$ vaccine through conjunctival route (MFAL, 2012). In this context, this study aims to investigate the field safety and efficacy of reduced doses of conjunctival S19 vaccine before the start of the mass vaccination program.

\section{MATERIALS AND METHODS}

In this study, except the animals in the control group (n:9), a total of 81 cattle aged from 3 months to 11 years old including 
pregnant ( $\mathrm{n}: 15)$ and lactating ( $\mathrm{n}: 42)$ animals were vaccinated by a reduced dose of $S 19$ vaccine through conjunctival route. One reduced dose of conjunctival S19 vaccine contains 5$10 \times 10^{9} \mathrm{cfu} /$ dose.

The study was conducted in Veterinary Control Institute after the necessary permission was obtained from the General Directorate of Food and Control of Ministry of Agriculture and Forestry related to field trials of conjunctival anti-brucella vaccines in cattle, which was dated 28.10.2010 and numbered B.12.0.KKG.0.19/108-02/15-2468-48891025366.

There were 15 pregnant and 42 lactating cattle in the vaccinated group. This field study was carried out in 7 farms in 4 different provinces. The animals in the vaccinated group were randomly selected. Unvaccinated animals in the herds were considered as the control group. The field conditions and rearing practices should be the same as much as possible (VICH, 2008) for animals belonging to both groups. Parallel to this, animals in similar herd conditions were used in this study. All the animals in this study were identified and monitored according to their ear tag number.

Vaccine product, which is the important component of the field studies, should belong to two or more production batches as representing the investigating vaccine $(\mathrm{VICH}$, 2008; OIE 2018b). In this study 3 production batches namely BA-K/09/01 BA-K/09/02 and BA-K/09/03 of BRUPEN-A conjunctival vaccines were used. Reduced dose of conjunctival S19 vaccine from these batches was $8.9 \times 10^{9}$, $9.1 \times 10^{9}, 8.1 \times 10^{9} \mathrm{cfu} /$ dose, respectively, which is in line with the reduced dose range of the vaccine.

All the procedures included in the safety and efficacy trials were carried out according to the rules outlined in manuals (EMEA, 2001; OIE, 2018b). In this study, the general health status of the animals during 21 days was observed to see if there is a systemic or local unexpected reaction after the first administration of the vaccine. Field safety trials focus on the presence of both local and systemic reactions such as allergic reactions, mortality, fever (EMEA, 2001). Vaccinated animals were re-vaccinated 4-6 months after the first vaccination as demonstrated in previous studies for conjunctival vaccination in cattle (Plommet et al., 1976; Nicoletti, 1978). A total of 4 animals from 3 different farms were vaccinated with an overdose of the vaccine. The body temperature of the animals randomly selected from control and vaccinated groups was recorded on the first 3 days after the vaccination. In addition, the effects of single, repeated and, overdose administration were examined based on the manual (OIE, 2018b).

2 weeks after the vaccination, nasal and conjunctival samples were taken from vaccinated animals representing $10 \%$ of the whole population. Milk samples were collected from lactating animals in the next 3 months following the vaccination and they were bacteriologically examined. Vaginal swab, colostrum and milk samples were taken from vaccinated pregnant animals 3 months after delivery at 2-week intervals. When there was no isolation of Brucella spp. from a sample after a 6 -week incubation period, that sample was regarded as negative (OIE, 2018a).

One of the main parameters of field efficacy trials is the indicators for a serological response. Within the scope of field efficacy trials, blood samples were collected on days $0,30,60,90$ in the post-vaccination period from vaccinated animals representing different ages and physiological conditions to evaluate humoral immune response. After the second vaccination (as a booster dose) of the same animals, the same procedure was followed for 4 months more, which makes 210 days post-vaccination period in total. Blood samples of the unvaccinated control group were also collected.

Blood samples were tested by Rose Bengal Plate Test (RBPT), Serum Agglutination Test (SAT) and Complement Fixation Test (CFT) as previously described (Alton et al., 1988). An animal showing 30 or more $\mathrm{IU} / \mathrm{ml}$ was accepted as positive in SAT. On the other hand, sera giving 20 International CFT Unit/ml titre or more were regarded as positive for CFT (OIE 2018a). Milk samples collected for 3 months after vaccination from lactating animals in different farms were analyzed via Milk Ring Test (MRT) for the detection of the antibodies in the milk. MRT enables the control of the larger amount of samples including pools of milk (Nielsen, 2002).

\section{RESULTS AND DISCUSSION}

We did not observe any local or systemic reactions in the animals throughout the study. This result is in line with previous studies (Beckett and MacDiarmid 1985, 1987; Kumar et al., 2016). In spite of the already existent studies, there was still a requirement of adequate data from our field conditions while changing the route and dose of the vaccine before the beginning of the mass vaccination program.

The results of conjunctival and nasal swab inoculations on Brucella selective medium are given in Table 1. The results were expressed as colony-forming units after the 15day incubation period.

The results showed that the existence of vaccine strain on ocular and nasal mucosa for only vaccinated group lasted for a few days. Vaccine strain isolation was not observed at the end of 15 days. No vaccine-induced abortion was observed in any of the 15 pregnant animals. No vaccine strain isolation was observed in the post-delivery vaginal swab, colostrum and milk samples of pregnant animals in the study. It is necessary to control several animal diseases with the help of vaccination to create an immunized host population. Therefore, only vaccines, which are considered to be safe and effective, could be utilized for the control and maintenance of animal health (OIE, 2018b).

Unlike the pregnant animals in this study, isolation of vaccine strain occurred in one sample among the milk samples taken from lactating animals during 3 months in the post-vaccination period. This isolation took place in the first month of this 3-month period and it did not continue afterwards. Vaginal swab and milk samples were also 
negative for the animals in the control group. Possible antibrucella antibodies were investigated in milk samples by MRT. The results of MRT are shown in Table 2.

The positivity rate of MRT at the end of 3 months postvaccination decreased to $5.7 \%$. When we evaluate the immune response detected from sera and milk, the results of MRT showed that there was a shorter response detected and a lower level of antibody titre in milk samples. This result is an expected scenario because milk without an infection might not include a high antibody titre. Indeed, the milk sample in which vaccine strain isolation was carried out has also a positive MRT result and it lasted positive at +++ level for 3 months. Regarding the results, there was more rapid clearance of antibodies in the milk than in blood samples. In this respect, preferring MRT in a dairy herd as a screening test might be useful for monitoring the disease. IndirectELISA and MRT are considered to be useful screening tests to monitor dairy cattle by using milk samples (OIE, 2018a). The humoral immune response detected by 3 serological tests after the first and the second vaccination was shown in Tables 3 and 4, respectively.

All the animals belonging to the control group remained seronegative from the beginning to the end of the study. Blood samples belonging to Farm-Il were found to be seropositive in the pre-vaccination period and the results of these animals were not included in the average results shown in Table 3 and 4 . The serological results of Farm-II after the first and the second vaccination were shown in Table 5 and 6 , respectively.
A high positivity rate for the humoral immune response was obtained from the first blood samples. However, when the positivity threshold was taken into account, the proportion of positive results is not very high. However, cell-mediated immunity takes an important role in protecting from brucellosis; therefore, the negative humoral response never excludes the possibility of being immune to the disease (Nicoletti, 1990; Schurig et al., 2002). The results of serological tests revealed that conjunctival vaccination with reduced dose induced short term antibody persistence without high antibody titres. The persistence of immune response was found to be 8 weeks after two vaccinations in a previous study (Plommet et al., 1976), which was slightly shorter than that of our results. Therefore, vaccination with a lower dose by conjunctival route was recommended to achieve a short-lived antibody response (Simpson, 2018). This advantage of the conjunctival route vaccination and reduced dose application may support mass vaccination programs by reducing the drawbacks of post-vaccinal serological responses.

Advantages of reduced dose conjunctival vaccination were also pointed out by other previous studies (Nicoletti et al., 1978; Jimenes de Bagues et al., 1991). Previous studies, in which standard and reduced doses of S19 vaccine were compared, revealed that the reduced dose was not only effective but it also led to a decline in the persistence of antibody titre (Alton et al., 1980; Alton and Corner 1981; Kumar et al., 2016) and vaccine-induced infections in pregnant cattle (Alton et al., 1980). The main factor behind

Table 1: Post-vaccination results of conjunctival and nasal swabs.

\begin{tabular}{lcccccc}
\hline Swab samples & $1^{\text {st }}$ day CFU & $2^{\text {nd }}$ day CFU & $3^{\text {rd }}$ day CFU & $7^{\text {th }}$ day CFU & $10^{\text {th }}$ day CFU & $15^{\text {th }}$ day CFU \\
\hline Conjunctival & 9.2 & 6.4 & 3.8 & 2.4 & 1.1 & 0 \\
Nasal & 5.8 & 4.2 & 2.1 & 0.9 & 0 & 0 \\
\hline
\end{tabular}

Table 2: Post-vaccination results of MRT in lactating cattle.

\begin{tabular}{lcccrrr}
\hline Post vaccination period & Negative & +++ & ++ & + & 0 & 0 \\
\hline Day 0 & 35 & 0 & 6 & 2 & 3 & 1 \\
1 month & 23 & 2 & 1 & 0 & 1 \\
2 months & 31 & 1 & 1 & 0 & 0 \\
3 months & 34 & 0 & 0 & 0 & $11.4 \%$ \\
4 months & 35 & $0.7 \%$ & 1 \\
\hline
\end{tabular}

Table 3: Post vaccination serological test results following the first vaccination.

\begin{tabular}{lcccccccccc}
\hline & \multicolumn{3}{c}{$1 \mathrm{MPV}$} & \multicolumn{3}{c}{ 2 MPV } & MPV \\
\cline { 2 - 8 } & RBPT & SAT & CFT & RBPT & SAT & CFT & RBPT & SAT & CFT \\
\hline Positivity rate $\%$ & 72.3 & 60.4 & 17.9 & 52.5 & 42.8 & 6.3 & 24.3 & 14.6 & 4.9 \\
\hline
\end{tabular}

${ }^{*}$ MPV- Month post vaccination.

Table 4: Post vaccination serological test results following the second vaccination.

\begin{tabular}{|c|c|c|c|c|c|c|c|c|c|c|c|c|}
\hline & \multicolumn{3}{|c|}{$1 \mathrm{MPV}$} & \multicolumn{3}{|c|}{$2 \mathrm{MPV}$} & \multicolumn{3}{|c|}{$3 \mathrm{MPV}$} & \multicolumn{3}{|c|}{$4 \mathrm{MPV}$} \\
\hline & RBPT & SAT & CFT & RBPT & SAT & CFT & RBPT & SAT & CFT & RBPT & SAT & CFT \\
\hline Positivity rate \% & 68.8 & 52.5 & 22.4 & 49 & 34.3 & 0 & 14.4 & 22.8 & 0 & 0 & 8.3 & 0 \\
\hline
\end{tabular}

*MPV- Month post vaccination. 
Investigating Field Efficacy and Safety of Conjunctival Brucella abortus S19 Vaccine in Cattle

Table 5: Post vaccination serological test results of farm II following the first vaccination.

\begin{tabular}{lcccccccccccc}
\hline FARM II & \multicolumn{3}{c}{ Day 0 } & \multicolumn{3}{c}{ 1 MPV } & \multicolumn{3}{c}{2 MPV } & \multicolumn{3}{c}{3 MPV } \\
\cline { 2 - 23 } & RBPT & SAT & CFT & RBPT & SAT & CFT & RBPT & SAT & CFT & RBPT & SAT & CFT \\
\hline Positivity rate $\%$ & 28.6 & 28.6 & 14.3 & 85.7 & 50 & 28.6 & 50 & 42.8 & 14.3 & 57.1 & 35.7 & 14.3 \\
\hline
\end{tabular}

*MPV- Month post vaccination.

Table 6: Post vaccination serological test results of Farm II following the second vaccination.

\begin{tabular}{|c|c|c|c|c|c|c|c|c|c|c|c|c|}
\hline \multirow{2}{*}{ FARM II } & \multicolumn{3}{|c|}{$1 \mathrm{MPV}$} & \multicolumn{3}{|c|}{$2 \mathrm{MPV}$} & \multicolumn{3}{|c|}{$3 \mathrm{MPV}$} & \multicolumn{3}{|c|}{$4 \mathrm{MPV}$} \\
\hline & RBPT & SAT & CFT & RBPT & SAT & CFT & RBPT & SAT & CFT & RBPT & SAT & CFT \\
\hline Positivity rate \% & 92.8 & 57.1 & 28.6 & 85.7 & 42.8 & 21.4 & 71.4 & 35.7 & 7.1 & 57.1 & 28.6 & 7.1 \\
\hline
\end{tabular}

${ }^{*} \mathrm{MPV}$ - Month post vaccination.

this is to receive a low number of bacteria resulting in a short immune response (Kumar et al., 2016).

The protection by low dose conjunctival S19 vaccines was also demonstrated similar or even better results with those of standard dose in guinea pigs (Plommet and Plommet 1975). The results of another study carried out with buffaloes revealed that the immune response and the duration depend on the dose of the S19 vaccine. In addition to this, an $\mathrm{S} 19$ vaccine containing $4 \times 10^{9} \mathrm{cfu} / \mathrm{dose}$, which is similar to the reduced dose, is recommended to get adequate protection for buffalo calves (Shome et al., 2020).

Almost all of the vaccinated 3-8 month calves turned out negative in all serological tests at the end of 8 weeks after vaccination. On the other hand, the positive results of older cattle such as heifers and cows lasted for 12 weeks. Moreover, even at the end of 16 weeks, positive test results from a few animals were still observed. These results indicated that there was a positive correlation between the host's age and the persistence of the humoral immune response. It was found out that calfhood vaccination induced a significant decrease in the percentage of the seropositive animals by CFT after adult vaccination (Beckett and Mac Diarmid 1985). None of the animals having an adult vaccination following the calfhood vaccination remained seropositive for two years after adult vaccination (Beckett and Mac Diarmid 1987).

Previous studies revealed that administration of a reduced dose of the $\mathrm{S} 19$ vaccine by conjunctival route does not create less protection than applying a higher dose by the subcutaneous way (Nicoletti et al., 1978; Nicoletti 1984). This route of vaccination with a reduced dose was thought to build adequate herd immunity (Jimenes de Bagues et al., 1991). Repeated conjunctival vaccination has an important advantage to be applied at any age without interfering significantly with serology. In addition to this advantage, it was indicated that this repeated administration would provide efficacy as much as the standard vaccination (Plommet et al., 1976).

Different administration routes such as subcutaneous, intradermal, conjunctival were evaluated previously and it was found out that the administration route had a slight effect on the efficacy of the vaccine (Nicoletti 1984). Therefore, the conjunctival administration route and reduced dose of
S19 vaccine followed in this study might be a suitable combination by providing the necessary efficacy and less serological interference during mass vaccination programs.

Even though the antibody titres were found to be higher after the second vaccination, there was not a significant difference between the two post vaccinations. Infected animals showed higher antibody titres and longer antibody latency after vaccination. The seropositivity rate of Farm II showed higher percentages than other farms based on three serological tests which were performed 4 months after the second vaccination. It is an expected result that the vaccination of animals, which had previous exposure to the pathogen, creates a stronger secondary immune response with a longer persistence as a booster effect. Therefore, it should be taken into consideration that serological responses detected in herds including infected animals might last longer following the vaccination.

Antibody titres and the persistence detected by the serological tests carried out in this study were compared and positive test results obtained from RBPT and SAT lasted longer with greater titres than CFT results. Positive results obtained thorough SAT sustained with an $8.3 \%$ positivity rate while other tests gave negative results 4 months after the second vaccination in this study. The CFT results are in line with those of the previous studies in which there was no seropositivity detected by CFT at the end of 4 months after vaccination (Jimenes de Bagueset al., 1991). It was pointed out that the vaccine dose had an effect on falsepositive test results. In addition, even if the standard dose or reduced dose were utilized, SAT and card test presented greater antibody levels and longer antibody persistence than rivanol or CFT tests (Nicoletti, 1984).

Two main characteristics of an ideal vaccine are its safety and its protective effect (Lalsiamthara and Lee, 2017). Vaccine efficacy and immunization coverage are the key factors to finalize the vaccination program successfully. Prevention of serious epidemic actually depends upon the high level of vaccine efficacy and coverage (Asokan, 2009). It should also be taken into consideration that the effectiveness of a vaccine in the target hosts is diversified by several factors such as feeding, environmental stress, vaccination dose, administration route, immunization and disease prevalence in herds (Schurig et al. 2002; Shome et al., 2020). 


\section{CONCLUSION}

In conclusion, a reduced dose of B.abortus $\mathbf{S 1 9}$ vaccine administered via conjunctival route is considered to be safe and it reduces the risk of vaccine-induced abortion and excretion from vagina and milk. With these advantages, it does not pose any threat to public health. It can be used as a suitable vaccination strategy for cattle against brucellosis in our field conditions in which subcutaneous vaccination with standard dose was applied for many years and it is particularly compatible with control programs for brucellosis. The findings of this study are considered to be significant to see the possible variations unique to this study's field conditions and the herd status before the mass vaccination.

\section{REFERENCES}

Alton, G.G. and Corner, L.A. (1981). Vaccination of heifers with a reduced dose of Brucella abortus strain 19 vaccine before first mating. Australian Veterinary Journal. 57(12): 548-50.

Alton, G.G., Corner, L.A. and Plackett, P. (1980). Vaccination of pregnant cows with low doses of Brucella abortus strain 19 vaccine. Australian Veterinary Journal. 56(8): 369-72.

Alton, G.G., Jones L.M., Angus, R.D. and Verger, J.M. (1988). Techniques for the Brucellosis Laboratory, Paris, France. Institut National de la Recherche Agronomique.

Asokan, G.V. (2009). Epidemiological assessment of vaccine efficacy Veterinary World. 2(3): 118-22.

Beckett, F.W. and MacDiarmid, S.C. (1985). The effect of reduceddose Brucella abortus strain 19 vaccination in accredited dairy herds. British Veterinary Journal. 141(5): 507-14.

Beckett, F.W. and MacDiarmid, S.C. (1987). Persistent serological titres following reduced dose Brucella abortus strain 19 vaccination. British Veterinary Journal. 143(5): 477-79.

Briones, G., Inon de lannino, N., Roset, M., Vigliocco, A., Paulo, P.S. and Ugalde, R.A. (2001). Brucella abortus cyclic beta-1, 2-glucan mutants have reduced virulence in mice and are defective in intracellular replication in HeLa cells. Infection and Immunity. 69: 4528-35.

Das, A., Kumar, B., Chakravarti, S., Pal Singh, K., Abhishek and Shrinet, G. (2019). Single-tube duplex-PCR for specific detection and differentiation of Brucella abortus S19 vaccine strain from other Brucella spp. Indian Journal of Animal Research. 53: 821-6.

EMEA, (2001). The European Agency for the Evaluation of Medicinal Products Committee for Veterinary Medicinal Products. EMEA/CVMP/852/99-FINAL, Note for Guidance Field Trials with Veterinary Vaccines.

Grillo, M.J., Bosseray, N. and Blasco, J.M. (2000). In Vitro Markers and Biological Activity in Mice of Seed Lot Strains and Commercial Brucella melitensis Rev 1 and Brucella abortus B19 Vaccines. Biologicals. Journal of the International Association of Biological Standardization. 28: $119-27$.

Godfroid, J., Cloeckaert, A., Liautard, J.P., Kohler, S., Fretin, D., Walravens, K., Garin-Bastuji, B. and Letesson, J.J. (2005). From the discovery of the Malta fever's agent to the discovery of a marine mammal reservoir, brucellosis has continuously been a re-emerging zoonosis. Veterinary Research. 36: 313-26
Jimenez de Bagues, M.P., Marin, C. and Blasco, J.M. (1991). Effect of antibiotic therapy and strain 19 vaccination on the spread of Brucella melitensis within an infected dairy herd. Preventive Veterinary Medicine. 1: 17-24.

Kumar, A., Verma, A.K., Prasad, V.S., Srinivas, K. and Reddy, G.S. (2016). Safety and efficacy of reduced dose Brucella abortus strain-19 vaccine in non-pregnant zebu cattle (Bos indicus) heifers. Indian Journal of Animal Science. 86(2): 136-39.

Lalsiamthara, J. and Lee, J.H. (2017). Development and trial of vaccines against Brucella. Journal of Veterinary Science. 18(S1): 281-90

MFAL, Ministry of Food, Agriculture and Livestock, General Directorate of Food and Control. (2012). Control and Eradication Project of Brucella with Conjunctival Vaccine, Circular No: 2012/03.

Naveen Kumar, V., Bharathi, M.V., Selvaraju, K., Porteen, K. and Vijayarani, K. (2019). Serum based polymerase chain reaction and enzyme linked immunosorbent assays for diagnosis of bovine brucellosis. Indian Journal of Animal Research. 53: 661-6.

Nicoletti, P. (1984). Vaccination of cattle with Brucella abortus strain 19 administered by differing routes and doses. Vaccine. 2: $133-135$

Nicoletti, P. (2010). Brucellosis: past, present and future. Prilozi. 31(1): 21-32.

Nicoletti, P., Jones, L.M. and Berman, D.T. (1978). Comparison of the subcutaneous and conjunctival route of vaccination with Brucella abortus strain 19 vaccine in adult cattle. Journal of the American Veterinary Medical Association. 173(11): 1450-1456.

Nicoletti, P. (1990). Vaccination. Animal Brucellosis. [(Eds) Nielsen, K. and Duncan, J.R.]. CRC Press, Boca Raton, Fla. pp. 284-99.

Nielsen, K. (2002). Diagnosis of brucellosis by serology. Veterinary Microbiology. 90(2002): 447-59.

OIE, World Organisation for Animal Health. (2018a). Terrrestrial Manual. Chapter 1.1.8 Principles of Veterinary Vaccine Production.

OIE, World Organisation for Animal Health. (2018b). Terrestrial Manual. Chapter 3.1.4. Brucellosis (Brucella abortus, B. melitensis, B. suis) (Infection with B. abortus, B. melitensis, B. suis)

Pappas, G., Panagopoulou, P., Chistou, L. and Akritidis, N. (2006). Brucella as a biological weapon. Cellular and Molecular Life Sciences. 63: 2229-36.

Plommet, M., Plommet, A.M. (1975). Vaccination against bovine brucellosis with a low dose of strain 19 administered by the conjunctival route. I-Protection demonstrated in guinea pigs. Annales de RecherchesVeterinaires. 6(4): 345-56.

Plommet, M. and Plommet, A.M. (1976). Vaccination against bovine brucellosis with a low dose of strain 19 administered by the conjunctival route. II-Determination of the minimum dose leading to colonization of the regional lymph nodes of cattle. Annales de RecherchesVeterinaires. 7(1): 1-8.

Plommet, M., Fensterbank, R. and Sauriau, A. (1976). Vaccination against bovine brucellosis with a low dose of strain 19 administered by the conjunctival route. III. -Serological response and immunity in the pregnant cow. Annales de Recherches Veterinaires. 7(1): 9-23. 
Ravi Kumar, D., Mishra, S.K., Kumar, A., Vineeth M.R., Jayakumar, S., Sakthivel Selvan, A., Karuthadurai, T., Chaudhuri, P., Kataria, R.S., Niranjan, S.K. (2019). Major histocompatibility complex (DRB3) gene expression pattern indicates differences in Brucella abortus $\mathrm{S} 19$ vaccine induced immune response in Karan Fries and Sahiwal cattle. Indian Journal of Animal Research. 53(2): 156-60.

Schurig, G.G., Sriranganathan, N. and Corbel, J.M. (2002). Brucellosis vaccines: Past, present and future. Veterinary Microbiology. 90: 479-96.

Seleem, M.N., Boyle, S.M. and Sriranganathan, N. (2010). Brucellosis: A re-emerging zoonosis. Veterinary Microbiology. 140(34): 392-98.

Simpson, G.J.G., Marcotty, T., Rouille, E., Chilundo, A., Letteson, J.J. and Godfroid, J. (2018). Immunological response to Brucella abortus strain 19 vaccination of cattle in a communal area in South Africa. Journal of the South African Veterinary Association. 89(0): a1527.
Shome, R., Kilari, S., Sahare, A., Kalleshamurthy, T., Heballi Niranjanamurthy, H., Ranjan Shome, B., Hiremath, J., Misri, J. and Rahman, H. (2020). Evaluation of the immune responses against reduced doses of Brucella abortus S19 (calfhood) vaccine in water buffaloes (Bubalus bubalis), India. Vaccine. 38(45): 7070-78.

Songer, J.G. and Post, K.W. (2012). Brusella cinsi. Veteriner Hekimlik Mikrobiyolojisi-Hayvan Hastalığı Etkeni Olan Bakteriler ve Mantarlar. [(Eds) Ang O and Ozgur Y.] Nobel Publishing, Istanbul, Turkey. pp. 200-207.

$\mathrm{VICH}$, International Cooperation on Hormonisation of Technical Requirements for Registration of Veterinary Medicinal Products. (2008). Guideline, VICH GL 44, Target Animal Safety for Veterinary Live and Inactivated Vaccines.

Wareth G. (Coord.) (2019). Brucellosis in the Mediterranean countries: History, prevalence, distribution, current situation and attempts at surveillance and control. OIE Technical Series Volume 12. pp. 98.

Yumuk, Z. and O'Callaghan, D. (2012). Brucellosis in Turkey-an overview. International Journal of Infection Disease 16(4): 228-35. 